\title{
Road to identifying endpoint biomarkers in the treatment of nonalcoholic steatohepatitis: are we there yet?
}

\author{
Donghee Kim $^{1}$, Luis Miguel Vazquez-Montesino², Aijaz Ahmed ${ }^{1}$ \\ ${ }^{1}$ Division of Gastroenterology and Hepatology, ${ }^{2}$ Department of Medicine, Stanford University School of Medicine, Stanford, CA, USA \\ Correspondence to: Aijaz Ahmed, MD. Division of Gastroenterology and Hepatology, Stanford University School of Medicine, 750 Welch Road, \# 210, \\ Palo Alto, CA 94304, USA. Email: aijazahmed@stanford.edu. \\ Provenance and Peer Review: This article was commissioned by the Editorial Office, Hepatobiliary Surgery and Nutrition. The article did not undergo \\ external peer review.
}

Submitted Sep 23, 2019. Accepted for publication Oct 09, 2019.

doi: 10.21037/hbsn.2019.10.02

View this article at: http://dx.doi.org/10.21037/hbsn.2019.10.02

In the United States (US), the landscape of chronic liver disease has been in flux in recent years as chronic hepatitis $\mathrm{C}$ virus (HCV) infection is no longer the leading indication for liver transplantation and now ranks behind alcoholic liver disease (ALD) and nonalcoholic steatohepatitis (NASH) as the third leading indication for liver transplantation (1). The approval of secondgeneration direct-acting antiviral (DAA) agents in late 2013 heralded a revolutionary era in the treatment of $\mathrm{HCV}$ infection. In a recent population-based study, there was a marked increase in age-standardized mortality for $\mathrm{HCV}$ infection with annual percent change (APC) of $2.2 \%$ in the pre-DAA era [2007-2014], but a marked decline in age-standardized mortality rates for HCV in the DAA-era [2014-2017] with -6.5\% of APC (2). While HCV-related mortality declined along with the introduction of DAA therapies, nonalcoholic fatty liver disease (NAFLD)-related death was on the rise at an accelerated pace in the US adults (3). Notably, age-standardized mortality for NASHrelated cirrhosis increased significantly between 2007 and 2016 (APC: 15.4\%; 95\% CI: 14.1 to 16.7 ) (4). NAFLDrelated advanced fibrosis remains a topic of interest because of its association with end-stage liver disease and hepatocellular carcinoma. During the last 10 years from 2005 to 2016, NAFLD-related advanced fibrosis increased from 3\% among subjects with NAFLD in 2005-2008 to $6 \%$ in 2013-2016 in the US adults (5). The prevalence of NAFLD-related advanced fibrosis is now estimated to be four million US adults (5). Longitudinal cohort studies looking into patients with NAFLD suggest that the fibrosis stage, and not the presence of NASH, predicted both the risk of overall and cause-specific mortality $(6,7)$. Therefore, NASH with presence of fibrosis has been identified as a measurable model for pharmacotherapy in the setting of a clinical trial (8). Cheung et al.'s recently published review article on the treatment trial endpoint for NASH and fibrosis is a timely discussion on this topic (8). This review article by The Liver Forum, a collaboration of academic investigators, professional societies, patient representatives, industry stakeholders and regulatory authorities is a comprehensive review of serum- and imaging-based markers that are potential surrogate markers of improvement in liver histology and are likely to reflect clinically meaningful assessment in a noninvasive fashion (8). According to US and European regulatory agencies, drug approval requires significant evidence of clinical benefit to patients, including a significant improvement in symptoms, functional capacity, or survival $(8,9)$. However, a significant number of patients with NASH are either asymptomatic or present with nonspecific symptomatology, which poses a substantial challenge in objective assessment of clinical improvement. Therefore, efforts and focus have shifted to develop and validate surrogate endpoint markers that predict a clinical benefit by preventing, retarding and reversing the progression NAFLD-related outcomes (8). The ideal surrogate marker(s) should be sensitive enough to reflect a clinically meaningful benefit for patients with both early and advance stages of NASH—a challenge $(8,9)$. Currently, improvement in fibrosis without worsening of NASH or resolution of NASH without worsening of fibrosis or combined improvement in fibrosis and resolution of NASH have been acknowledged as meaningful histologic 
endpoints for NASH in phase III clinical trials $(8,9)$. Due to shortcomings of histological assessment, including sampling variability, intra- and interobserver variation, and misinterpretation for the use of numerical descriptors for fibrosis severity, and invasive nature of liver biopsy, there is a clear need to investigate and identify serumand/or imaging-based surrogate markers/biomarkers. The magnitude of change in the serum- and/or imaging-based biomarkers during treatment should predict prevention, reduction and/or reversal of clinical outcomes.

\section{Assessment of change in steatosis}

Change in liver fat content is currently not valid as an endpoint for the phase III clinical trial. However, in the phase IIa trials where evidence of potential benefit is sought without histologic confirmation, evaluation of change in steatosis may have a role as an endpoint, along with additional markers reflective of improved liver injury such as decline in the liver enzyme (8). Several studies showed that the reduction in liver fat content parallels with histologic improvements $(10,11)$. Currently, magnetic resonance imaging (MRI)-proton-density-fat-fraction (PDFF) may be more sensitive and precise than histologic grading for assessing changes in liver fat content, although the magnitude of change that correlates with meaningful outcomes needs further validation.

\section{Assessment of changes in NASH}

For the diagnosis of NASH, liver biopsy remains the irreplaceable gold standard. Other imaging methods lack sufficient accuracy and sensitivity to differentiate between nonalcoholic fatty liver (NAFL) and NASH. Magnetic resonance elastography (MRE) is used to determine liver stiffness; interpretation of MRE changes must take into account the effects of both fibrosis and inflammation. Multiparametric MRI is another investigative test that provides a continuous Liver Inflammation and Fibrosis (LIF) score that is proposed to correlate with the severity of NASH (12). Additional serum markers and panels require further validation and changes in these panels will likely be evaluated in the ongoing phase II and phase III trials.

\section{Assessment of changes in fibrosis}

Serum markers and panels that have been studied or validated in patients with NAFLD include the aspartate aminotransferase to platelet ratio index (APRI), FIB-4 scores, and NAFLD fibrosis score, etc. When advanced fibrosis was evaluated by noninvasive NAFLD fibrosis score, APRI, and FIB-4 score, all-cause mortality was more frequent in the intermediate and high probability of advanced fibrosis than in the low probability of advanced fibrosis which is mainly derived from cardiovascular causes, independent of other known factors (7). However, due to limited current evidence, the utility of these noninvasive serum panels to evaluate changes in treatment response will need to be further validated. While liver stiffness measurements by fibroscan was overestimated in NAFLD, this problem can be partly improved using the XL probe (13). MRE can assess the entire liver and is more accurate than fibroscan for identifying the stage of fibrosis, but its limited availability and higher cost may be impediments to widespread use (14).

\section{Changes in functional testing}

While functional testing is a novel approach to assess the severity and changes in actual liver function, functional testing has been challenging because the multiple liver functions may not change in parallel with the progression of liver disease and due to inter-variability between the tests (8). The HepQuant ${ }^{\circledR}$ test and methacetin breath test reflect the metabolic capacity of the liver, which remain to be validated in future studies $(8,15)$.

\section{Conclusions}

As the field of pharmacologic treatment for NASH expands, systemic analysis of samples acquired by randomized controlled trials may lead to the identification and validation of broad-spectrum surrogate markers/biomarkers as measures of treatment response and assessment of end points. Although the final goal is to identify panel noninvasive markers/biomarkers which will provide the versatility to assess and predict progression, retardation and reversal of NASH-related clinical outcomes and hepatic histological damage. We have made a significant amount of progress, but we are not there yet.

\section{Acknowledgments}

Funding: None. 


\section{Footnote}

Conflicts of Interest: All authors have completed the ICMJE uniform disclosure form (available at https://hbsn. amegroups.com/article/view/10.21037/hbsn.2019.10.02/coif). The authors have no conflicts of interest to declare.

Ethical Statement: The authors are accountable for all aspects of the work in ensuring that questions related to the accuracy or integrity of any part of the work are appropriately investigated and resolved.

Open Access Statement: This is an Open Access article distributed in accordance with the Creative Commons Attribution-NonCommercial-NoDerivs 4.0 International License (CC BY-NC-ND 4.0), which permits the noncommercial replication and distribution of the article with the strict proviso that no changes or edits are made and the original work is properly cited (including links to both the formal publication through the relevant DOI and the license). See: https://creativecommons.org/licenses/by-nc-nd/4.0/.

\section{References}

1. Cholankeril G, Ahmed A. Alcoholic Liver Disease Replaces Hepatitis C Virus Infection as the Leading Indication for Liver Transplantation in the United States. Clin Gastroenterol Hepatol 2018;16:1356-8.

2. Kim D, Adejumo AC, Yoo ER, et al. Trends in Mortality From Extrahepatic Complications in Patients With Chronic Liver Disease, From 2007 Through 2017. Gastroenterology 2019;157:1055-66.e11.

3. Kim D, Li AA, Gadiparthi C, et al. Changing Trends in Etiology-Based Annual Mortality From Chronic Liver Disease, From 2007 Through 2016. Gastroenterology 2018;155:1154-63.e3.

4. Kim D, Li AA, Perumpail BJ, et al. Changing Trends in Etiology- and Ethnicity-Based Annual Mortality Rates of Cirrhosis and Hepatocellular Carcinoma in the United States. Hepatology 2019;69:1064-74.

5. Kim D, Kim W, Adejumo AC, et al. Race/ethnicitybased temporal changes in prevalence of NAFLD-related advanced fibrosis in the United States, 2005-2016. Hepatol Int 2019;13:205-13.

6. Dulai PS, Singh S, Patel J, et al. Increased risk of mortality by fibrosis stage in nonalcoholic fatty liver disease: Systematic review and meta-analysis. Hepatology
2017;65:1557-65.

7. Kim D, Kim WR, Kim HJ, et al. Association between noninvasive fibrosis markers and mortality among adults with nonalcoholic fatty liver disease in the United States. Hepatology 2013;57:1357-65.

8. Cheung A, Neuschwander-Tetri BA, Kleiner DE, et al. Defining Improvement in Nonalcoholic Steatohepatitis for Treatment Trial Endpoints: Recommendations from the Liver Forum. Hepatology 2019;70:1841-55.

9. Sanyal AJ, Friedman SL, McCullough AJ, et al. Challenges and opportunities in drug and biomarker development for nonalcoholic steatohepatitis: findings and recommendations from an American Association for the Study of Liver Diseases-U.S. Food and Drug Administration Joint Workshop. Hepatology 2015;61:1392-405.

10. Patel J, Bettencourt R, Cui J, et al. Association of noninvasive quantitative decline in liver fat content on MRI with histologic response in nonalcoholic steatohepatitis. Therap Adv Gastroenterol 2016;9:692-701.

11. Ajmera V, Park CC, Caussy C, et al. Magnetic Resonance Imaging Proton Density Fat Fraction Associates With Progression of Fibrosis in Patients With Nonalcoholic Fatty Liver Disease. Gastroenterology 2018;155:307-10.e2.

12. Pavlides M, Banerjee R, Tunnicliffe EM, et al. Multiparametric magnetic resonance imaging for the assessment of non-alcoholic fatty liver disease severity. Liver Int 2017;37:1065-73.

13. Vuppalanchi R, Siddiqui MS, Van Natta ML, et al. Performance characteristics of vibration-controlled transient elastography for evaluation of nonalcoholic fatty liver disease. Hepatology 2018;67:134-44.

14. Kim D, Kim WR, Talwalkar JA, et al. Advanced fibrosis in nonalcoholic fatty liver disease: noninvasive assessment with MR elastography. Radiology 2013;268:411-9.

15. Helmke S, Colmenero J, Everson GT. Noninvasive assessment of liver function. Curr Opin Gastroenterol 2015;31:199-208.

Cite this article as: Kim D, Vazquez-Montesino LM, Ahmed A. Road to identifying endpoint biomarkers in the treatment of nonalcoholic steatohepatitis: are we there yet? HepatoBiliary Surg Nutr 2020;9(2):244-246. doi: 10.21037/hbsn.2019.10.02 